\title{
Perioperative consideration of general anesthesia for acromegalic patients
}

\author{
Seunghyun Kang, Yong-Hyun Cho, Sun-Hee Kim, and Dong-Hyun Lee \\ Department of Anesthesiology and Pain Medicine, Seoul Sungsim General Hospital, Seoul, Korea
}

Acromegaly is a condition caused by an excessive secretion of growth hormones [1]. Hypertrophy in the face and extremities can cause enlarged lower jaws, macroglossia, and thickened pharyngeal and laryngeal soft tissues [2]. Hypertrophy in the vocal cord can lead to changes in the upper airway, making airway management difficult during trachea intubation [3,4]. For 50\% of acromegalic patients, collateral circulation of radial artery and ulnar artery is not clearly visible in one hand or both hands [5]. Therefore, catheter should be carefully inserted into the radial artery in the wrist to continuously measure the blood pressure. We report a case of successful general anesthesia for an acromegalic patient.

A 63 year-old male patient diagnosed with ossification of yellow ligament T11-12 and spinal stenosis L1-5 was hospitalized for an operation of posterolateral fusion T11-12 and decompression L1-5. Two years ago, a macroadenoma of $17 \times 27 \mathrm{~mm}$ was observed in the patient by sella magnetic resonance imaging at another hospital. The height of the patient was $200 \mathrm{~cm}$. His weight was $94 \mathrm{~kg}$. Based on results of laboratory examination and chest $\mathrm{x}$-ray performed before surgery, nospecific abnormal finding was observed. Electrocardiogram (ECG) revealed signs of AV block type I. Physical examination found clinical signs of Mallampati class III, jaw malocclusion, and macroglossia. After performing Allen's test, flushing did not occur in the left handeven in 15 seconds. Flushing in the right hand was delayed for 9 seconds. In the following upper extremity computed tomography angiography (CTA), collateral blood flow of radial and ulnar artery was not clearly visible (Fig. 1A).

No premedication was carried out. After the patient arrived in the operation room, routine monitoring tests such as non invasive blood pressure, ECG, and pulse oximetry were performed. His vital signs were stable, with blood pressure (BP)
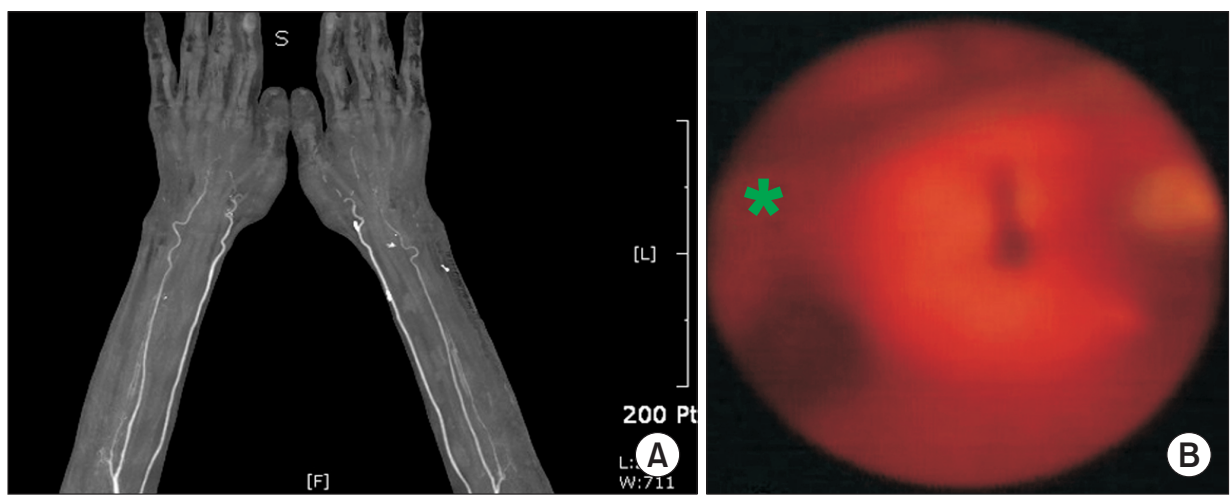

Fig. 1. Upper Extremity Computed Tomography Angiography (CTA). The collateral blood flow between radial and ulnar artery in both hands was not clearly visible (A). View of vocal cord by the Bullard laryngoscope showing a narrowed glottis due to hypertrophy in the patient with height of $200 \mathrm{~cm}$ and weight of $94 \mathrm{~kg}$ (B).

Corresponding author: Seunghyun Kang, M.D., Department of Anesthesiology and Pain Medicine, Seoul Sungsim General Hospital, 259, Wangsan-ro, Dongdaemun-gu, Seoul 130-867, Korea. Tel: 82-2-966-1616, Fax: 82-2-968-2394, E-mail: joyster21@gmail.com

(c) This is an open-access article distributed under the terms of the Creative Commons Attribution Non-Commercial License (http:// creativecommons.org/licenses/by-nc/3.0/), which permits unrestricted non-commercial use, distribution, and reproduction in any medium, provided the original work is properly cited. 
at $135 / 70 \mathrm{mmHg}$, heart rate $(\mathrm{HR})$ at 65 beats/min, and $\mathrm{SpO}_{2}$ at $99 \%$.

After preoxygenation with $100 \%$ oxygen $\left(\mathrm{O}_{2}\right), 0.2 \mathrm{mg}$ of glycopyrrolate was intravenously injected followed by $180 \mathrm{mg}$ of $1 \%$ propofol. When his unconsciousness was confirmed, $80 \mathrm{mg}$ of rocuronium was intravenously injected. After performing mask ventilation using oral airway, his vocal cord was observed using Bullard laryngoscope. His glottis became narrow (Fig. 1B). An armored tube with a diameter of $7.5 \mathrm{~mm}$ was intubated into the trachea. To continuously measure his BP, an invasive arterial catheterization was inserted into his dorsalis pedis artery in the left foot. His central venous pressure (CVP) was monitored using internal jugular vein catheterization. The operation lasted about 8 hours. Anesthesia was maintained with isoflurane at $1-1.5 \mathrm{vol} \%$ and remifentanil at $0.05 \mu \mathrm{g} / \mathrm{kg} / \mathrm{min}$ in an $\mathrm{O}_{2}-\mathrm{N}_{2} \mathrm{O}$ mixture at a $1: 1$ ratio. During surgery, his systolic BP was maintained at $105-135 \mathrm{mmHg}$, with diastolic BP maintained at $55-75 \mathrm{mmHg}$. His $\mathrm{HR}$ was $60-90$ beats/min, with $\mathrm{SpO}_{2}$ at $99 \%$ and CVP at 6-7 $\mathrm{cmH}_{2} \mathrm{O}$. After the surgery, the patient fully recovered spontaneous breathing and consciousness. Extubation was performed. The patient was in a stable condition after the surgery. He was later transferred to the surgical intensive care unit.

Upper airway changes often occur in acromegalic patients [2]. Due to changes in the upper airway, intubation can be difficult $[3,4]$. Preoperative examinations, such as head and neck movement, Mallampati classification, and thyromental distance, can predict the difficulty of intubation [3]. Our patient showed a good result of head and neck movement before surgery. However, he had Mallampati classification class III, $10 \mathrm{~cm}$ thyromental distance, macroglossia, and jaw malocclusion. Based on these findings, we predicted that intubation would be difficult. Therefore, we planned intubation using the Bullard laryngoscope. During intubation, it was confirmed that glottis was narrowed. An armored tube of $7.5 \mathrm{~mm}$ in diameter which was small for the patient's body size was inserted into the trachea. Since the intu- bation into the trachea of acromegalic patients is difficult, it is recommended to use Mackintosh Curve-shaped No. 5 blade or use other methods such as an intubating laryngeal airway mask and a light wand. Intubation into the trachea using fibroptic bronchoscope under consciousness is considered the safest way [4].

Radial artery, an invasive cannulation route in the wrist, is commonly used for continuous measurement of blood pressure. To make use of that route, the ulnar artery circulation to a hand should be checked for impairment first. If the radial artery cannulation is implemented without checking the ulnar artery circulation to a hand, it can cause ischemic damage to the hand. Allen's test can be used to detect impaired ulnar artery circulation to the hand. Acromegalic patients often have carpal ligament hypertrophy which leads to compression to the ulnar artery. Because of this, they can develop impaired ulnar artery circulation. Approximately $50 \%$ of acromegalic patients have impaired ulnar artery circulation in one or both of their hands, with $30 \%$ of those who have impaired ulnar artery circulation can develop carpal tunnel syndrome [5]. However, our patient did not exhibit any signs of carpal tunnel syndrome. Allent's test, which was performed before surgery, showed that flushing did not occur in the left hand even in 15 seconds, while the right hand exhibited a delayed response of 9 seconds. The upper extremity CTA did not clearly show the collateral blood flow of radial and ulnar artery in both hands. Therefore, it is necessary to secure a cannulation route into the dorsalis pedis artery for continuous monitoring of blood pressure.

The intubation into the trachea of an acromegalic patient can be difficult due to anatomical changes. Radial artery cannulation may require extra attention and care due to impaired ulnar artery collateral circulation. Therefore, an accurate medical history checkup of related symptoms as well as strict evaluations and necessary tests must be performed before surgery. An adequate anesthesia plan must be set up and implemented.

\section{References}

1. Scacchi M, Cavagnini F. Acromegaly. Pituitary 2006; 9: 297-303.

2. Melmed S. Medical progress: Acromegaly. N Engl J Med 2006; 355: 2558-73.

3. Schmitt H, Buchfelder M, Radespiel-Tröger M, Fahlbusch R. Difficult intubation in acromegalic patients: incidence and predictability. Anesthesiology 2000; 93: 110-4.

4. Nemergut EC, Zuo Z. Airway management in patients with pituitary disease: a review of 746 patients. J Neurosurg Anesthesiol 2006; 18: 737.

5. Campkin TV. Radial artery cannulation. Potential hazard in patients with acromegaly. Anaesthesia 1980; 35: 1008-9. 